\title{
Numerical study of wet plastic particle separation using a coupled DEM-SPH method
}

\author{
Darius Markauskas ${ }^{\mathrm{a}, *}$, Harald Kruggel-Emden ${ }^{\mathrm{b}}$, Viktor Scherer ${ }^{\mathrm{a}}$ \\ ${ }^{a}$ Ruhr-University Bochum, Universitaetsstrasse 150, D-44780 Bochum, Germany \\ ${ }^{b}$ Technical University of Berlin, Ernst-Reuter Platz 1, D-10587 Berlin, Germany
}

\begin{abstract}
The separation of different kind of plastic particles is required in the process of waste recycling. For the separation drum processes passed through by a liquid are applicable. Thereby the separation is based on the principle that particles either sink or float in a liquid depending on their densities. In this study the aforementioned process is numerically analysed for the separation of polyethylene terephthalate (PET) from polypropylene (PP) particles. The Discrete Element Method coupled with the Smoothed Particle Hydrodynamics method (DEM-SPH) is used for modelling purposes. The employment of the SPH for the modelling of the liquid let us exploit the strong side of this meshless method, namely, the relative easiness to model large movements of the fluid together with free surfaces and moving boundaries. The used theoretical model is presented and validation tests are performed, where a dam-break problem is considered as an example. Simulations of the plastic particle separation in the rotating drum are performed thereafter. The influence of the different operational and design parameters, such as the rotational velocity, the feed rate, the number of lifters etc., on the resultant purity of the plastic is estimated. It is expected that in the future the performed analysis will allow to optimise drum separation processes.
\end{abstract}

Keywords: solid-liquid flow, wet particle separation, fluid-particle interaction, discrete element method, smoothed particle hydrodynamics

\section{Introduction}

Mechanical plastic recycling is currently one of the weakest steps in the recycling system, because only a low percentage of plastic is reused compared to the amount of recovered metal, glass and waste paper [1]. As the raw mixture of plastic waste usually includes various kinds of plastics (e.g. Acrylonitrilebutadiene-styrene (ABS), Polyethylene terephthalate (PET), Polystyrene (PS), Polyethylene (PE), Polypropylene (PP), Polyvinyl chloride (PVC)), the separation process should classify waste into a number of reclaimable plastic fractions, so as to meet the requirements for the purity and cleanliness of a polymer type that are needed in a high quality plastic recycling process [2].

* Corresponding author

Email address: markauskas@leat.rub.de, Tel: +49 (0) 234 / 32-27281, Fax: +49 (0) 234 / 32-14227 (Darius Markauskas) 

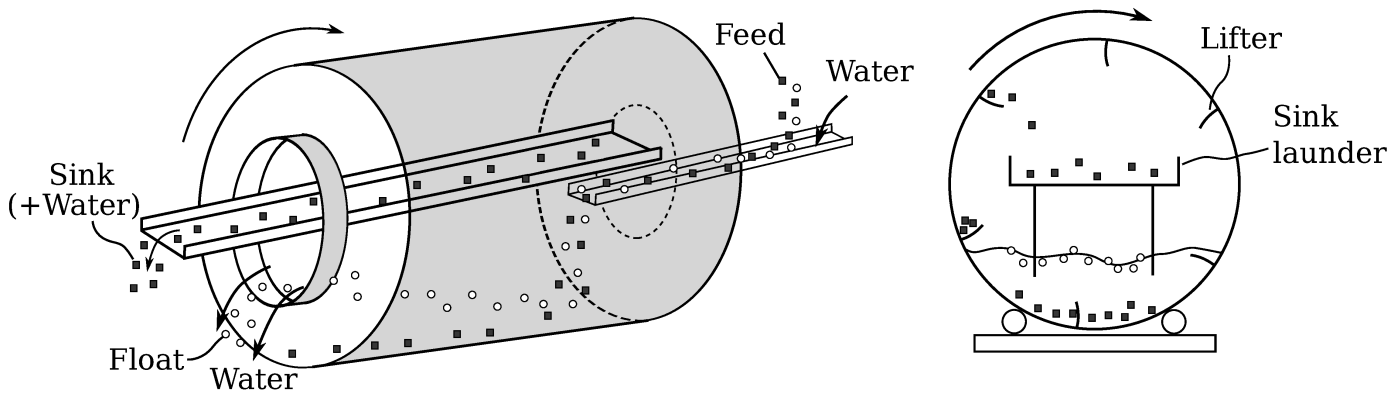

Figure 1: Scheme of a drum separator

Mechanical plastic recycling processes can be divided into wet and dry separation techniques. Among the wet processes drum separators based on the float-sink principle [3] are widely used for separating granular materials (Fig.11). They are based on the fact that grains either sink or float in liquids depending on their densities. Before the separation process, the plastic wastes are shredded into small particle-like entities. The resulting plastic particle mixture is fed together with water into a rotating drum. The sinking particles are lifted onto a sink launder and are then removed from the drum, while the floating particles are discharged out of the vessel together with the water. To shift the cut point to a different density, it may be necessary to vary the density of the separating liquid. This is done either by dissolving materials of lower or greater density than water, for example alcohols or salts, or by suspending fine grained solids of greater density than water. The latter separating liquid is also known as a heavy medium and is extensively employed in mineral processing industry [4].

The successful separation of binary plastic mixtures in rotating drums was studied experimentally by Dodbiba et al. [1]. A review of varying separation technologies and their efficiencies was compiled by the same authors [5]. For multi-component plastic mixtures a three stage processes [6] was proposed which results in good recovery rates for most kinds of plastic. Difficulties arise in floatsink separation if density differences between plastic fractions become low or in case of elevated feed rates [7].

Potentially, the numerical modelling could contribute for the improvement of wet separation of particles. Thereby for modelling of plastic particles, a Discrete Element Method (DEM) can be used. DEM was first proposed by Cundall and Strack in 1979 and since then is widely used in many areas of powder technology and mechanical process engineering [8, 9]. In the DEM, solid particles are considered to be separate entities, interacting with each other by contacts mostly. This discrete character of the method allows a reduced set of constitutive assumptions to be used as compared to continuum approaches.

By modelling of a mixed fluid-particle system, a coupling between solid particles and fluid is required. In the coupled system, the fluid phase can be modelled at the sub-particle level such that momentum exchange between fluid and particle is resolved in detail 10], or using local averaging techniques 11]. The simulations at the sub-particle level allow a detailed analysis of interaction forces, that act between the fluid and solid particles, and, therefore, can be used, e.g. for determination of drag correlations [12]. However, such simulations are computationally very expensive and, therefore, they usually are limited to a 
small number of solid particles [13, 14]. The use of local averaging techniques is computationally more efficient and allows simulations of much larger particle systems, while preserving the discrete characteristics of the particle flow.

For the modelling of the fluid flow, Navier-Stokes equations are usually solved using mesh-based methods, like Finite Volume or Finite Element Methods 15]. However, the application of mesh based methods for modelling of complex geometries and free surfaces, can be a challenging task [16]. To elevate these difficulties a Smoothed Particle Hydrodynamics (SPH) method can be used. The SPH, originally proposed by Gingold and Monaghan [17] and Lucy [18], is a mesh-less Lagrangian technique, which proved to be a suitable tool for modelling of fluids in such areas as marine [19], extrusion [20], geophysical [21] or costal 22] engineering. The major strength of this method is its mesh-less character, which makes the method very flexible and enables the simulation of engineering problems, that might be difficult to capture by conventional mesh-based methods.

Recently, the two-way coupling between DEM and SPH based on a local averaging technique was proposed by Gao and Herbst [16], Sun et al. [23] and Robinson et al. [24]. The successful application of the two-way coupled DEMSPH to slurry flow, abrasive wear and magnetorheological fluids was demonstrated by Cleary [25], Beck \& Eberhard [26] and Lagger et al. 27] respectively. A detailed analysis of the sedimentation of one particle and a porous block presented in 24] and a comparative study on coupling the DEM with meshbased methods and DEM coupling with the mesh-less SPH method reported by Markauskas et al. 28] allow to conclude, that DEM-SPH is an appropriate and promising tool for modelling particle-laden fluid systems.

In the current investigation the wet separation of plastic particles in a rotating drum is analysed numerically. The two-way coupled DEM-SPH method is used for this purpose. In our earlier work [28] the study on the coupling was presented, while in the current investigation, the earlier developed famework is applied to a real engineering problem. The flexibility of the DEM-SPH method enables us to simulate rapid movements of the particle-laden fluid with free surfaces and moving walls, and allows to analyse the influence of various operational and design parameters on the separation process. To our best knowledge, the wet particle separation in a rotating drum is analysed numerically for the first time.

\section{Governing equations}

\subsection{Governing equations of the solid phase}

The discrete element method is used for modelling of the solid particles. The motion of each particle is governed by Newton's second law:

$$
m_{i} \frac{d \mathbf{u}_{i}}{d t}=\mathbf{F}_{i}^{c}+\mathbf{F}_{i}^{g}+\mathbf{F}_{i}^{i n t},
$$

where $\mathbf{u}_{i}$ is the solid particle velocity, $\mathbf{F}_{i}^{c}$ is the contact force, $\mathbf{F}_{i}^{g}$ is the gravity force. $\mathbf{F}_{i}^{\text {int }}$ is the interaction force acting between solid and fluid phase. The details how this force is calculated is given in Section 2.3. The contact force for 
particle $\mathcal{P}_{i}$ is obtained as a sum of all contact forces between $\mathcal{P}_{i}$ and particles in contact $\mathcal{P}_{j}$ :

$$
\mathbf{F}_{i}^{c}=\sum_{j=1}^{n} \mathbf{F}_{i j}^{c},
$$

where $n$ is the number of contacts. For the calculation of the contact force in the normal direction a Hertz contact model together with the damping model developed by Tsuji et al. (1992) [29] are used. In the tangential direction the force is described by a spring limited by the Coulomb law characterised by the coefficient of tangential friction [30]. In the present study, only spherical particles are considered. A more detailed description of the used DEM model can be found in [31, 32].

\subsection{Governing equations of the fluid phase}

The Smoothed Particle Hydrodynamics (SPH) method [17, 18] is used for modelling of the fluid and is coupled with the DEM. The SPH treats the fluid in a completly mesh-free fashion in terms of a set of sampling points (particles) 33]. SPH particles represent a finite mass of the discretized fluid and carry information about all physical variables evaluated at their positions. Hydrodynamic equations for motion are derived for these particles.

The continuity equation and the momentum equation in a Lagrangian framework take the form [24]:

$$
\begin{gathered}
\frac{D \bar{\rho}_{f}}{D t}+\nabla \cdot\left(\bar{\rho}_{f} \mathbf{u}_{f}\right)=0, \\
\frac{D \bar{\rho}_{f} \mathbf{u}_{f}}{D t}=-\nabla p+\nabla \cdot(\varepsilon \boldsymbol{\tau})-\mathbf{f}^{i n t}+\bar{\rho}_{f} \mathbf{g},
\end{gathered}
$$

where $\overline{\rho_{f}}=\varepsilon \rho_{f}$ is the superficial density of the fluid, $\varepsilon$ is the local mean fluid volume fraction, $\mathbf{u}_{f}$ is the fluid velocity, $p$ denotes the pressure, $\boldsymbol{\tau}$ is the viscous stress tensor, $\mathbf{f}^{\text {int }}$ is the interaction force between fluid and solid particles and $\mathrm{g}$ is the gravitational constant.

SPH particles carry variables such as velocity, pressure and mass. While no connectivity is modelled between the SPH particles, the function values are interpolated from the neighbouring particles using a smoothing kernel function. The kernel function is defined so that its value monotonously decreases as the distance between particles increases. The influence radius of the kernel function is defined by the smoothing length $h$. There are several kernel functions used in SPH such as the Gaussian [34], quadratic [35] or quintic spline [36]. In the current study a commonly used cubic spline kernel [37, 38] is utilised:

$$
W(r, h)=\alpha_{D} \begin{cases}1-\frac{3}{2} q^{2}+\frac{3}{4} q^{3}, & 0 \leq q<1 \\ \frac{1}{4}(2-q)^{3}, & 1 \leq q<2, \\ 0, & q \geq 2,\end{cases}
$$

where $q=r / h, \alpha_{D}=1 /\left(\pi h^{3}\right)$ for the $3 \mathrm{D}$ case, $h$ is the smoothing length, which defines the influence volume of the kernel and $r$ is the distance between the two points of interest. 
An equation of state is used to estimate the pressure from the density field in the weekly compressible SPH method [39, 33]:

$$
p=\frac{\rho_{0} c^{2}}{\gamma}\left[\left(\frac{\overline{\rho_{f}}}{\varepsilon \rho_{0}}\right)^{\gamma}-1\right],
$$

where $\rho_{0}$ is the initial density of the fluid phase and $c$ is the speed of sound. It is recommended to use $c=10 u$ to keep the density to vary by at most $1 \%$ [40, 39], where $u$ is the maximum fluid velocity magnitude. The coefficient $\gamma=7$ is commonly used in SPH. However we experienced numerical instabilities when fluid together with solid particles were considered in the simulations. Gao and Herbst [16] following Morris et al. [40] recommend to use $\gamma=1$ to avoid these numerical problems. Following this recommendation $\gamma=1$ is used in the present study.

The continuity equation (3) in the SPH takes the form

$$
\frac{D \bar{\rho}_{a}}{D t}=\sum_{b} m_{b} \mathbf{u}_{a b} \cdot \nabla_{a} W_{a b},
$$

where indexes $a$ and $b$ indicate fluid particles. $m$ is the mass. $\mathbf{u}_{a b}=\mathbf{u}_{a}-\mathbf{u}_{b}$ is the relative velocity between particles $\mathcal{P}_{a}$ and $\mathcal{P}_{b} . \nabla_{a} W_{a b}=\nabla_{a} W\left(r_{a}-r_{b}, h\right)$ is the gradient of the kernel function. $r_{a}$ and $r_{b}$ are positions of the fluid particles $\mathcal{P}_{a}$ and $\mathcal{P}_{b}$. The summation is performed over all neighbouring particles of particle $\mathcal{P}_{a}$.

The momentum conservation equation (4) in SPH takes the form [40]:

$$
\begin{aligned}
& \frac{D \mathbf{u}_{a}}{D t}=-\sum_{b} m_{b}\left(\frac{p_{a}}{\bar{\rho}_{a}^{2}}+\frac{p_{b}}{\bar{\rho}_{b}^{2}}\right) \nabla_{a} W_{a b}+\mathbf{g}+ \\
& +\sum_{b} m_{b} \frac{\nu\left(\bar{\rho}_{a}+\bar{\rho}_{b}\right)}{\bar{\rho}_{a} \bar{\rho}_{b}} \cdot \frac{\mathbf{r}_{a b} \nabla_{a} W_{a b}}{\left|\mathbf{r}_{a b}\right|^{2}+\delta^{2}} \mathbf{u}_{a b}+\frac{\mathbf{f}_{a}^{i n t}}{m_{a}}
\end{aligned}
$$

The third term on the right hand side in Eq. (8) is a viscous term introduced by Morris [40], where $\nu$ is the kinematic viscosity. $\delta$ is a small number used just to keep the denominator non-zero which here is set to $0.1 \mathrm{~h}$.

$\mathbf{f}_{a}^{\text {int }}$ in Eq. (8) is the solid-fluid interaction force acting on the fluid particle $\mathcal{P}_{a}$ due to the solid particles. The force $\mathbf{f}_{a}^{i n t}$ is calculated as the sum over all solid particles in the domain of the fluid particle:

$$
\mathbf{f}_{a}^{i n t}=\sum_{i}-\frac{V_{a} W_{a i}}{\sum_{b} V_{b} W_{b i}} \mathbf{F}_{i}^{i n t} .
$$

where $V_{a}$ is the volume of fluid particle, while $\mathbf{F}_{i}^{i n t}$ is the interaction force acting on the solid particle (see Eq. (1)).

The fluid volume fraction $\varepsilon_{a}$ of the fluid particle $\mathcal{P}_{a}$ is calculated from the volumes of all solid particles $\mathcal{P}_{i}$ which are in the smoothing domain of the fluid particle $\mathcal{P}_{a}$ :

$$
\varepsilon_{a}=1-\sum_{i} V_{i} W_{a i}
$$

where $V_{i}$ is the volume of the solid particle $\mathcal{P}_{i}$, while $W_{a i}=W\left(r_{a}-r_{i}, h\right)$ is the kernel function Eq. (5).

Fluid particles are moved using a velocity smoothed by the average in their 
neighbourhood according to the kernel function, i.e. XSPH variant introduced by Monaghan [Mon1989]:

$$
\frac{d \mathbf{r}}{d t}=\mathbf{v}_{a}+\varepsilon_{X S P H} \sum \frac{m_{b}}{\hat{\rho}_{a b}}\left(\mathbf{v}_{b}-\mathbf{v}_{a}\right) W_{a b},
$$

where $\varepsilon_{X S P H}$ is the parameter here used equal to 0.5 and $\hat{\rho}_{a b}=\left(\rho_{a}+\rho_{b}\right) / 2$. This smoothed particle velocity reduces the fluid particle disorder, while does not change the overall linear momentum.

An often discussed topic in the SPH literature is the use of boundary conditions [41, 42, 43]. It is related to the fact, that the description of boundaries in the SPH is not as straightforward as in other grid based CFD methods. There are several approaches to enforce no-penetration boundaries in the SPH, in which mostly special fluid wall particles are introduced. In our earlier study 28] a modification of a no-slip no-penetration boundary was proposed, in which instantaneously generated ghost-fluid particles were used. These boundaries performed well in the test cases in [28]. However in the current study, where particle separation in rotating drum is simulated, we experienced numerical problems when the fluid particles are moved above the free surface of the fluid by the lifters (Fig.(1). In this situation a negative pressure (tension) in some of the fluid particles arose, which caused an artificial attraction between those particles and the nearby walls. To prevent this problem, a repulsive force boundary model proposed by Monaghan [44] is used in the current study. For this boundary condition the force between the fluid particle and the wall does not depend on the fluid particle pressure. Therefore, no artificial tension between a particle and a wall is generated. The same type of boundary condition was used by Gao et al. [16] and Robinson et al. [24] in their studies applying the coupled DEM-SPH method.

\subsection{Fluid-solid interaction}

The interaction force acting on a solid particle $\mathbf{F}_{i}^{\text {int }}$ in this study is calculated as the sum of the drag force $\mathbf{F}_{i}^{D}$ and the pressure gradient force $\mathbf{F}_{i}^{\nabla p}$ :

$$
\mathbf{F}_{i}^{i n t}=\mathbf{F}_{i}^{D}+\mathbf{F}_{i}^{\nabla p} .
$$

In the current study the correlation proposed by Di Felice [45], which is well-anticipated in literature, is used for the calculation of the drag force:

$$
\mathbf{F}_{i}^{D}=\frac{1}{8} C_{d} \rho_{f} \pi d_{i}^{2}\left(\mathbf{u}_{f, i}-\mathbf{v}_{i}\right)\left|\mathbf{u}_{f, i}-\mathbf{v}_{i}\right| \varepsilon_{i}^{2-\chi}
$$

where $\varepsilon_{i}, d_{i}, \mathbf{u}_{f, i}, \mathbf{v}_{i}$ are the fluid fraction at the location of solid particle $\mathcal{P}_{i}$, the solid particle diameter, the fluid velocity and the solid particle velocity correspondingly. $\varepsilon_{i}$ is obtained from the fluid fractions at the surrounding fluid particles:

$$
\varepsilon_{i}=\frac{\sum_{a} \varepsilon_{a} V_{a} W_{a i}}{\sum_{a} V_{a} W_{a i}} .
$$

The drag coefficient $C_{d}$ and the coefficient $\chi$ are calculated as a function of the particle Reynolds number [45]. 
Assuming that the pressure gradient $\nabla p$ arises only because of the interaction between solid particles and fluid, $F_{i}^{D}$ can be combined with $F_{i}^{\nabla p}$ [46], which results in:

$$
\mathbf{F}_{i}^{i n t}=\frac{\mathbf{F}_{i}^{D}}{\varepsilon}-V_{i} \rho_{f} \mathbf{g} .
$$

$\mathbf{F}_{i}^{i n t}$ is used in Eq. (1) and Eq. (9). More details about the used DEM-SPH model can be found in [28].

\section{Verification of the numerical model}

For verification purposes of the theoretical model presented in section 2 , numerical tests of a dam break problem are performed. In subsection $3.1 \mathrm{a}$ single phase (liquid only) dam break problem, while in subsection 3.2 a two phase (liquid and solid particles) dam break problem are simulated and obtained results are compared with available results found in literature.

\subsection{Dam break: Single-phase test}

In this test, a part of a rectangular container is filled with water (Fig. 2). The width of the liquid column is $a=0.2 \mathrm{~m}$ and the height is $2 a=0.4 \mathrm{~m}$. The gravity force is acting downwards with the magnitude $a$, i.e. $0.2 \mathrm{~m} / \mathrm{s}^{2}$. At the start of the simulation, an initialisation step is performed, during which the liquid particles are allowed to reach a static equilibrium condition. Then the right wall of the container is removed and the liquid is flowing along the horizontal bottom plane as a consequence. The $2 \mathrm{D}$ numerical test thereby using the volume of fluid (VOF) method was initially performed and reported by Hirt and Nichols [47]. The same test was also used by Sun et al. [23]. Two simulations are performed: one using an initial distance between liquid particles equal to $a / 10$ and one using an initial distance between liquid particles equal to $a / 20$. Totally 3000 and $24000 \mathrm{SPH}$ particles are used for the first and for the second simulation respectively. Liquid particle positions for the $a / 20$ simulation at different time instances are shown in Fig. 2. The position of the leading edge of the liquid vs time is presented in Fig. 3. where SPH results performed with two different SPH particle resolutions together with VOF results from [47] can be compared.

It can be seen in Fig. 3, that when using $a / 10 \mathrm{SPH}$ particles, the difference between $\mathrm{VOF}$ and $\mathrm{SPH}$ results reaches $5 \%$ at $t \sqrt{2 g / a}=2.0$. The reduction of the size of the SPH particles by a factor of two $(a / 20)$ reduces this difference down to $1 \%$. The obtained results let us conclude, that the used SPH model reproduces VOF results very well.

\subsection{Dam break: Two-phase test}

A simulation of the dam break problem, in which water is laden with solid particles, is performed as a second test case. The obtained numerical results are compared with experimental results reported by Sun et al. [23]. A rectangular container with size $20 \mathrm{~cm} \times 10 \mathrm{~cm} \times 15 \mathrm{~cm}$ (see Fig.4) is divided into two sections (one larger and one smaller section) by a vertical wall. In a first step, solid particles are generated with fluid particles placed atop of them in the smaller section of the container (Fig. 4a). Spherical solid particles with 
a)

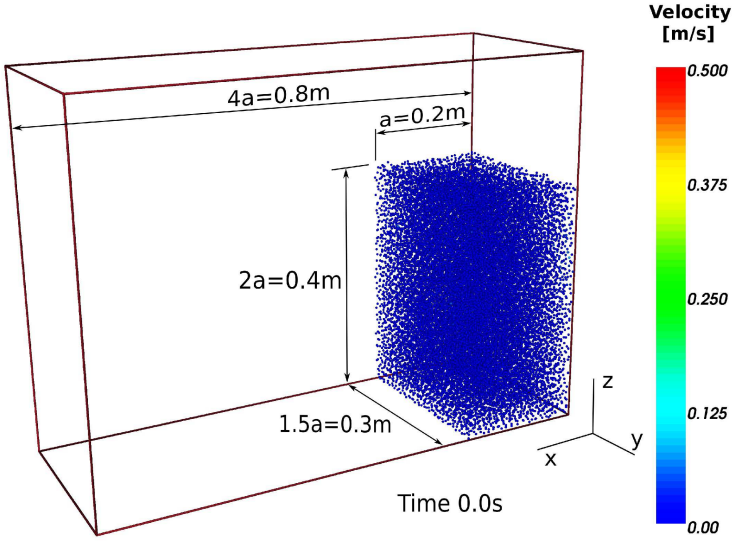

b)

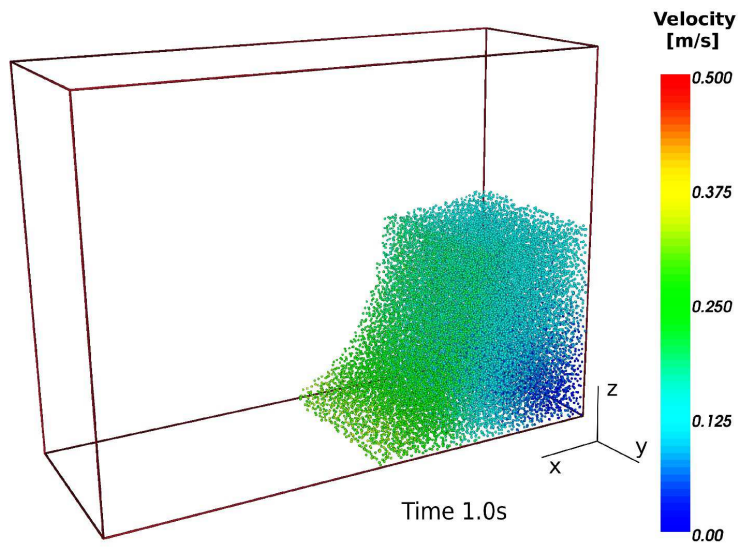

c)
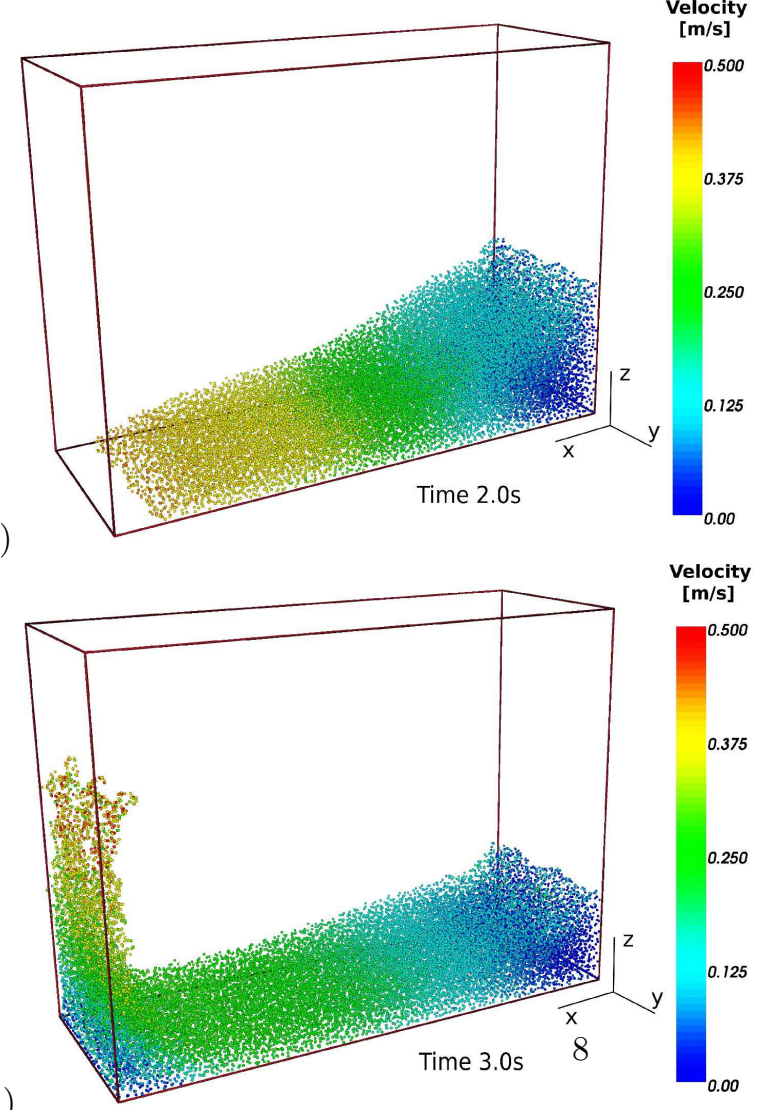

d)

Figure 2: SPH particles representing the liquid in the single-phase dam break test 


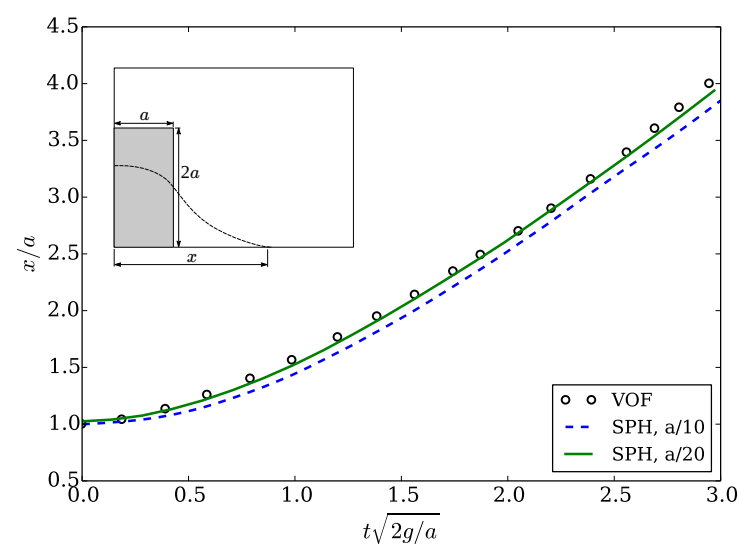

Figure 3: Position of the leading edge of the liquid in the single-phase dam break test

diameter $2.7 \mathrm{~mm}$ and density $2500 \mathrm{~kg} / \mathrm{m}^{3}$ are used for modelling of the glass beads. $200 \mathrm{~g}$ of solid material, which results in 7762 particles, is generated. The particle's Young's modulus equals $100 \mathrm{MPa}$, its Poisson's ratio is 0.2 , its restitution coefficient is set to 0.9 and the friction coefficient equals 0.2 as reported for the solid particles in the experiment [23]. For modelling of the water phase, which is used in 23. in the performed experiment, $5870 \mathrm{SPH}$ particles with a density of $\rho_{0}=1000 \mathrm{~kg} / \mathrm{m}^{3}$, a dynamic viscosity $\mu=0.001 \mathrm{~Pa} \cdot \mathrm{s}$, a smoothing radius $h=5.4 \mathrm{~mm}$ and an initial distance between SPH particles equal to $h / 1.3$ are used. An initialisation is performed during which the solid particles and the liquid are allowed to settle down by the action of gravity. In the actual simulation, the vertical wall (dam), which divides the container into two sections, is raised by a constant velocity of $v_{x}=0.68 \mathrm{~m} / \mathrm{s}$, therefore the solid particles-fluid mixture is moving out from the filled section. The change of the position of the leading edge of the fluid and the solid particles is shown in Fig.5.

As can be seen in Fig. [5 the simulation results match well with the experiment. Some minor divergence of the positions of the solid particles obtained numerically from the reported experimentally measured values at $t=0.16-0.20 \mathrm{~s}$ can be observed from the presented curves, however. The possible reason for this minor divergence could be in the physical properties of solid particles (restitution coefficient, friction coefficient) which where just roughly estimated in Ref. [23].

\section{Numerical analysis of wet plastic particle separation}

\subsection{Simulation setup and parameters}

A numerical analysis of the separation of plastic particles using a rotating drum is performed. The outline of the drum is presented in Fig.1 During the separation process the mixture of grains together with water is fed into the rotating drum through the opening on the right side (Fig.1 1). By interaction of gravity and buoyancy forces, the grains with a density lower than the liquid density are floating, while the grains with a density higher than the liquid density start to sink. The floating grains together with the liquid are discharged out through the opening on the left side of the drum. The sunken particles are lifted by lifters attached to the walls of the drum and dropped on the sink launder. 


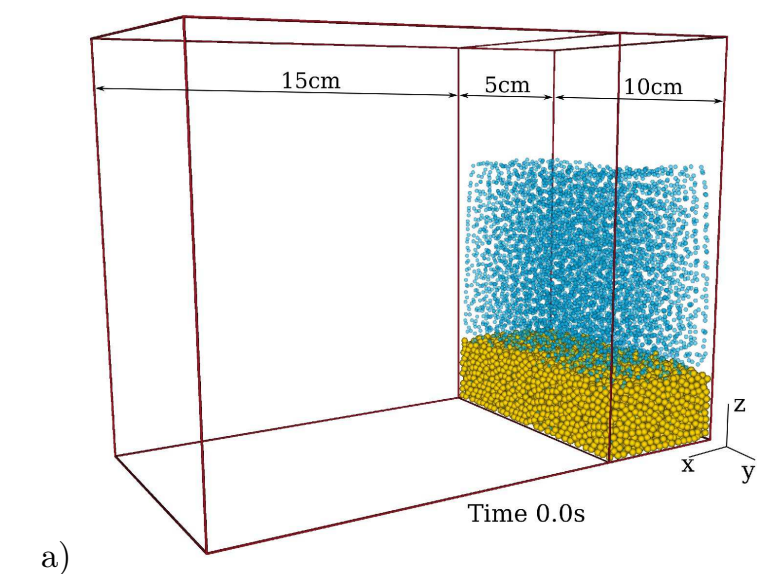

b)

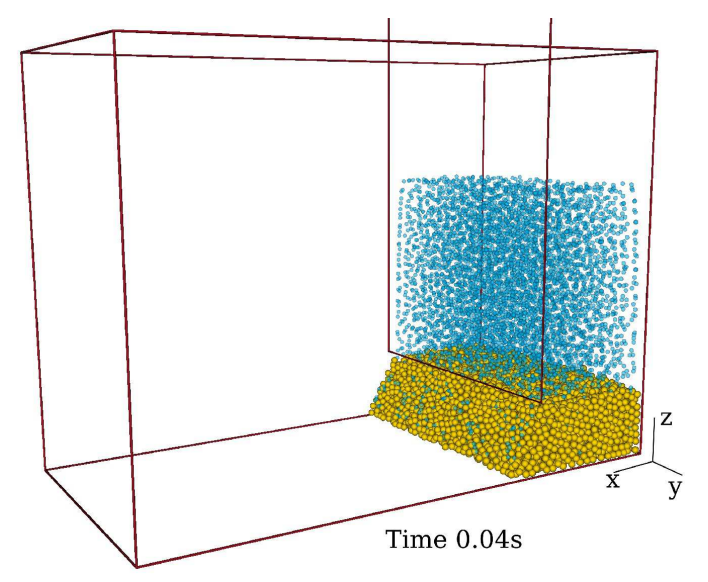

c)

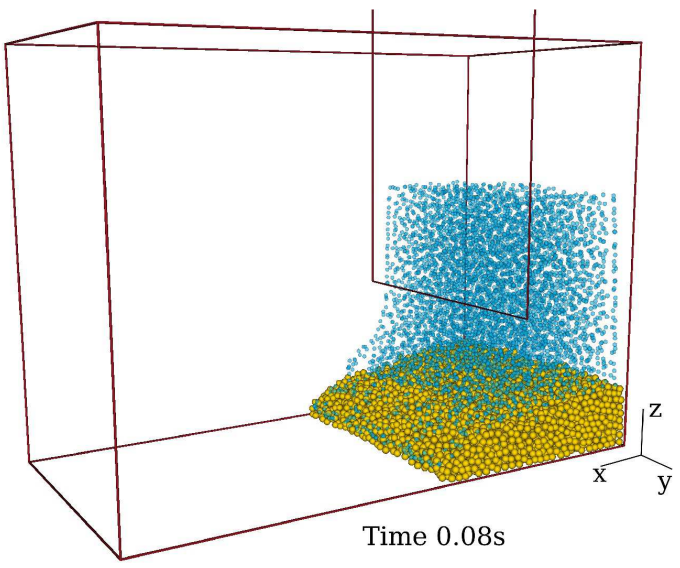

d)

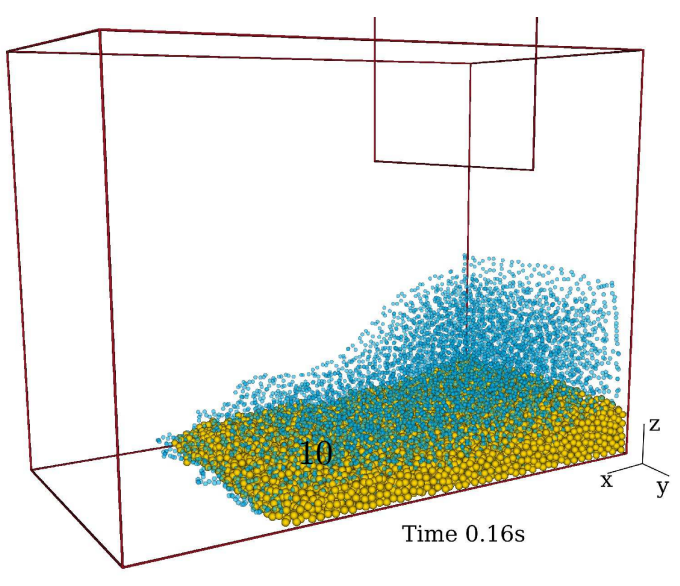

Figure 4: SPH particles (representing the liquid) and solid particles in the two-phase dam break test 


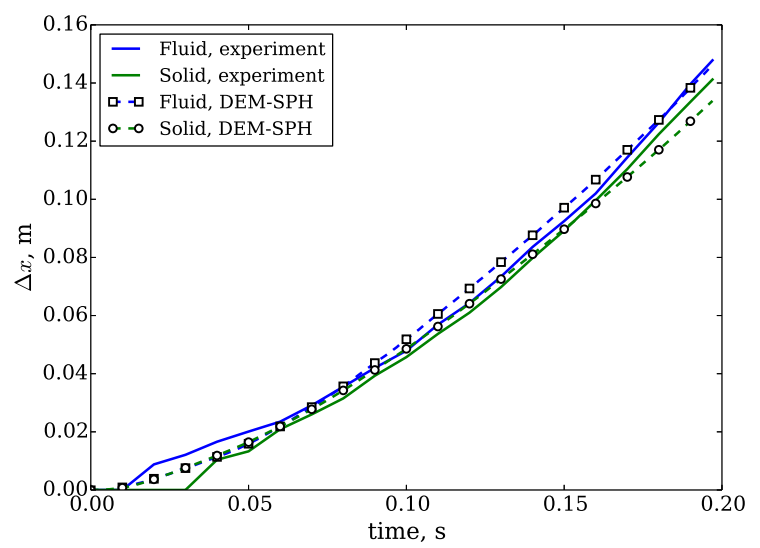

Figure 5: Temporal variation of the position of the leading edge of the the fluid and solid particles in the two-phase dam break test

The separation of polyethylene terephthalate (PET) from polypropylene (PP) is simulated. The density of PET particles is considered with $1350 \mathrm{~kg} / \mathrm{m}^{3}$ and the density of PP particles with $950 \mathrm{~kg} / \mathrm{m}^{3}$; a restitution coefficient of 0.5 is used. A density of $1000 \mathrm{~kg} / \mathrm{m}^{3}$ and a dynamic viscosity of $0.001 \mathrm{~Pa} \cdot \mathrm{s}$ are used for the liquid aligned with the properties of water.

In the simulations a simplified scheme of the laboratory scale drum shown in Fig.6 is used. Initially a prefill of the drum is numerically performed, where $0.0198 \mathrm{~m}^{3}$ of water are generated inside of the drum and a simulation of $1 \mathrm{~s}$ is performed during which this water settles and partly flows out of the drum forming a starting condition. Then the mixture of solid particles and fluid is started to be generated inside the drum in small chunks every $0.09 \mathrm{~s}$. By varying the size of the chunk a possible variation of the flow rate is achieved. In all performed simulations the particle mixture contains an equal volume of PET and PP material. For the modelling of the PET and PP material, spherical particles with random diameter between 3-4 mm are used. For the modelling of the water, SPH particles with a kernel length of $h=8 \mathrm{~mm}$ and an initial distance of $h / 1.3$ are utilised. The kernel length is chosen based on the simulation results described in [24] and the analysis of the settlement of a single particle in [28]. All simulations are performed until $40 \mathrm{~s}$ of operation time is reached. During the simulation the sinking/sunken solid particles are lifted from the bottom of the drum and are dropped into the "Sink Remove Zone" (SRZ) (see Fig.6), where particles are removed from the simulation. Thereby the discharge process of the sinking/sunken solid particles is represented in a simplified manner which however has no implication on the accuracy of the simulation. The floating solid particles together with the water flow out through the opening in the drum and are also removed ("Float Remove Zone" (FRZ) in Fig.6) .

Overall 10 simulations are performed with the purpose to analyse the sensitivity of the separation quality to different parameters. The considered parameters are given in Table1. As a base setup (BS in Table1) a simulation with a rotational velocity of the drum of $0.5 \pi \mathrm{rad} / \mathrm{s}, 4$ lifters, a solid feed rate of $29.3 \mathrm{~g} / \mathrm{s}$ and a water flow rate of $2.07 \cdot 10^{-3} \mathrm{~m}^{3} / \mathrm{s}$ is utilised. Also vertical walls inside of the drum (see Fig.6) are used to form a zone where the separation 


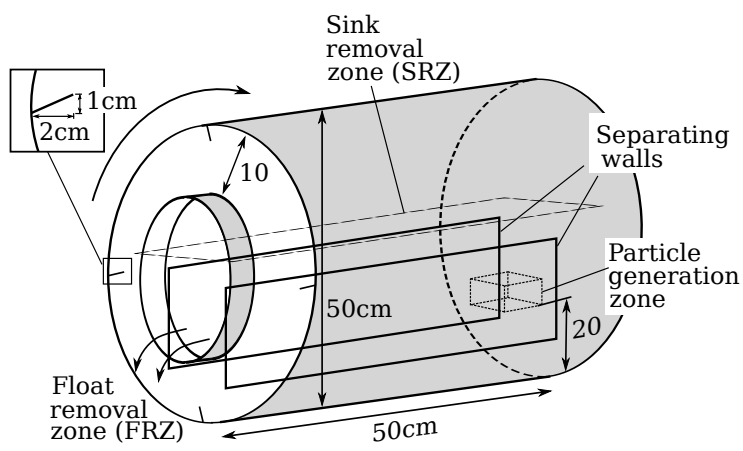

Figure 6: Scheme of the drum separator used in the simulations

Table 1: Simulation parameters for testing of the drum separation

\begin{tabular}{|c|c|c|c|c|c|}
\hline Label & Separating walls & $\begin{array}{c}\text { Rotational } \\
\text { velocity of the } \\
\text { drum, rad/s }\end{array}$ & $\begin{array}{l}\text { Number of } \\
\text { lifters }\end{array}$ & $\begin{array}{l}\text { Solid feed rate, } \\
\mathrm{g} / \mathrm{s}\end{array}$ & $\begin{array}{c}\text { Water flow rate, } \\
\mathrm{m}^{3} / \mathrm{s}\end{array}$ \\
\hline$\overline{B S}$ & Yes & 0.5 & 4 & 29.3 & $2.07 \cdot 10^{-3}$ \\
\hline $\mathrm{S} 1$ & No & & & & \\
\hline $\mathrm{S} 2$ & & $0.25 \pi$ & & & \\
\hline S3 & & $\pi$ & & & \\
\hline $\mathrm{S} 4$ & & & 2 & & \\
\hline S5 & & & 8 & & \\
\hline S6 & & & & 14.65 & \\
\hline S7 & & & & 58.6 & \\
\hline $\mathrm{S} 8$ & & & & & $1.035 \cdot 10^{-3}$ \\
\hline S9 & & & & & $4.14 \cdot 10^{-3}$ \\
\hline
\end{tabular}

of solid particles should take place. Every simulation S1-S9 differs from the BS just by one parameter with a two times smaller and two times larger value being applied. In Table1 only the parameters for the BS and the parameters for S1-S9, which differ from the BS, are presented.

\subsection{Simulation of basic setup}

Several snapshots of the simulation of the basic setup (BS in Table1) at different instances of time are presented in Fig.7. In the figures, the SPH water particles are shown in a light blue colour and are semi transparent. PET particles $\left(\rho=1350 \mathrm{~kg} / \mathrm{m}^{3}\right)$ are shown in a red colour and PP particles $(\rho=$ $950 \mathrm{~kg} / \mathrm{m}^{3}$ ) are represented dark blue. As already mentioned in Section 4.1 , the drum is initially filled with water. The column of water is generated inside of the drum at $t=0 \mathrm{~s}$ (Fig.7 $7 \mathrm{a}$ ), which is allowed to settle down until $1 \mathrm{~s}$ (Fig.7 b). Then the mixture of water and solid particles is started to be generated inside the drum. In Fig.7 $7 \mathrm{c}$ and Fig.7 d it can be seen how solid particles are moved up out of the liquid by the lifters. However not only sunken PET particles (red colour), but some water and floating PP particles (blue colour) are caught and transported to the Sink Remove Zone (SRZ) (see Fig.6). Here the fluid and liquid particles are removed from the simulation, which has no effect on the separation process as even in reality they would leave the system here. 


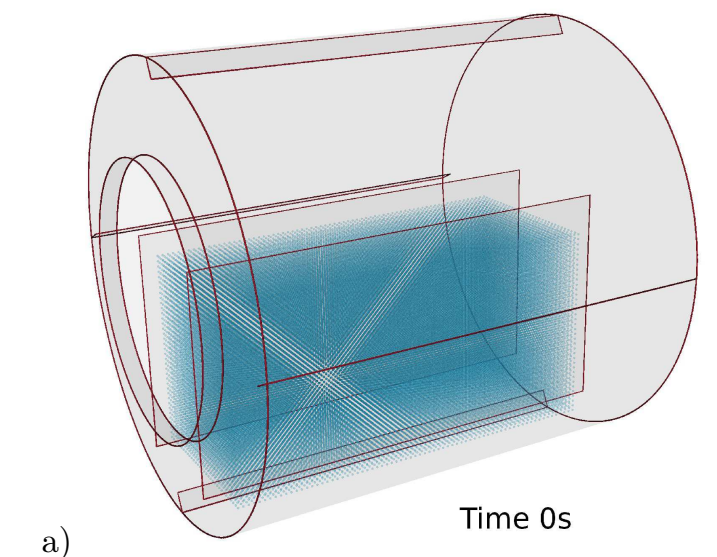

a)

Time 0s

b)

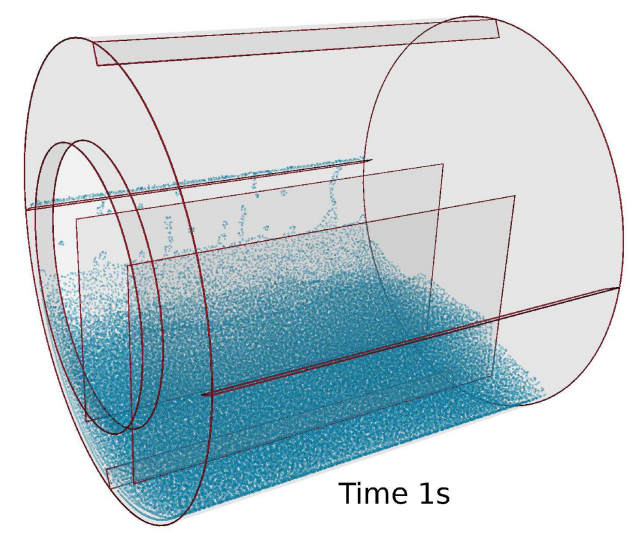

c)
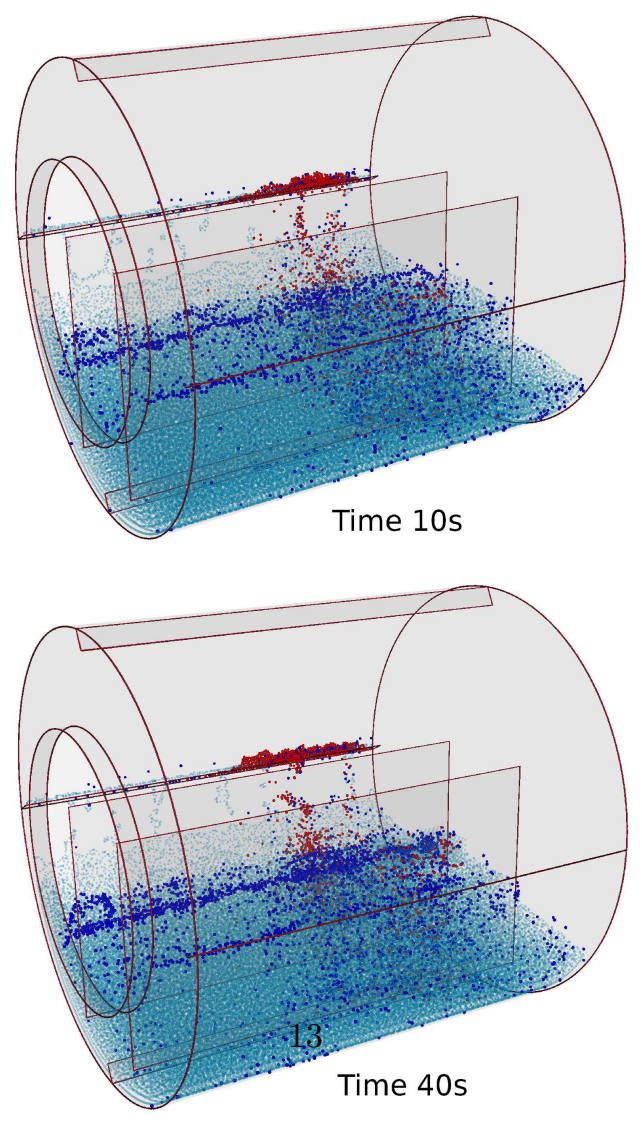

Figure 7: Simulation of particle separation in the rotating drum: snapshots taken at different time instances 


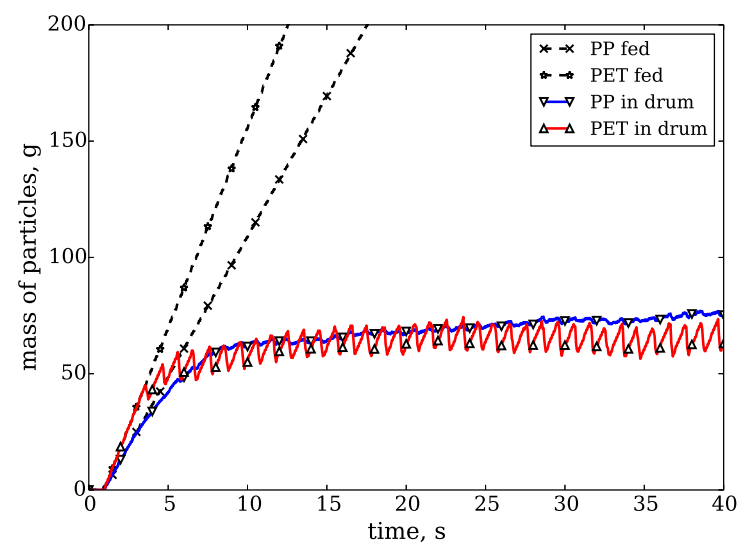

Figure 8: Basic setup (BS) simulation: the mass of the generated particles and the mass of the particles inside of the drum

The mass of the fed plastic particles and the mass of the plastic particles inside the drum during the simulation are shown in Fig. 8, While in the figure the shown mass of the fed particles is cut at $200 \mathrm{~g}$, the particles are charged constantly until the end of the simulation. The mass of PET particles inside of the drum at first increases rapidly, however from about $t=6 \mathrm{~s}$, the amount of PET particles inside remains almost unchanged. The stepwise character of the "PET in drum" curve reflects the time intervals at which the rotating lifters remove sunken PET particles. The amount of PP particles increases rapidly until about $t=8 \mathrm{~s}$, but then a light steady increase is forming out which remains until the end of the simulation.

From the amount of the removed particles a mass flow rate is calculated. Because particles can be removed either when they are lifted to the sink remove zone (SRZ), or when they float through the left opening in the drum (float remove zone, FRZ) (see Fig.66), two mass rates for every particle kind are obtained and shown in Fig.9. In an ideal separation case, all PET particles should reach the SRZ, while all PP particles should exit through the FRZ. While no PET particle is transported to the FRZ, in the basic simulation case (BS) some PP particles are moving into the SRZ together with PET. Therefore, the resulting "SRZ PP" curve is non-zero.

\subsection{Analysis of the influence of different design and operational parameters}

\subsubsection{Influence of separating walls}

The influence of separating vertical walls (see Fig.6) is analysed. In many drum separators they are used to confine the zone of the settling of particles. Besides the basic setup (BS) simulation, in which vertical walls are used, a simulation without vertical walls (S1 in Table1) is performed. The development of the mass flow rate of particle removal is shown in Fig.10, "S1 SRZ PET" and "BS SRZ PET" curves are always keeping at the same level during the simulation. However the "S1 SRZ PP" curve is always above the "BS SRZ PP" curve, which indicates, that in the $\mathrm{S} 1$ case more $\mathrm{PP}$ particles are lifted together with PET particles. In the last $15 \mathrm{~s}$ the mass flow rate of PP particles reaching the SRZ increases in the simulation without the vertical walls ("S1 SRZ PP" 


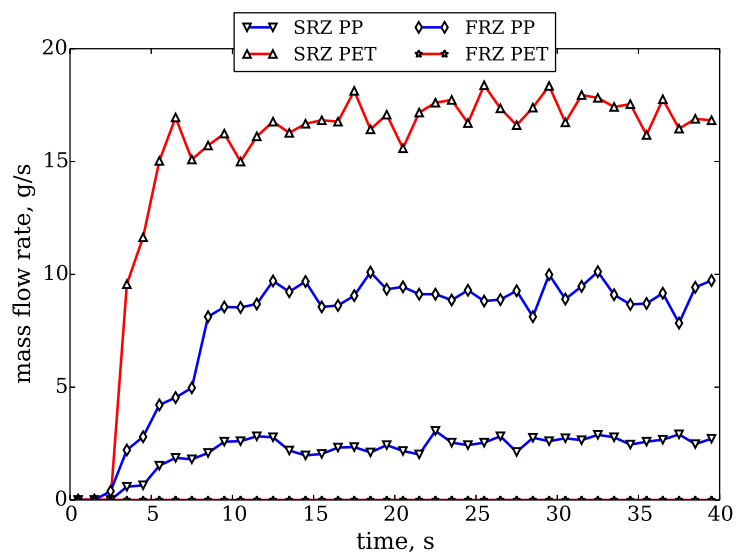

Figure 9: Mass flow rate of the separated particles in the basic simulation (BS)

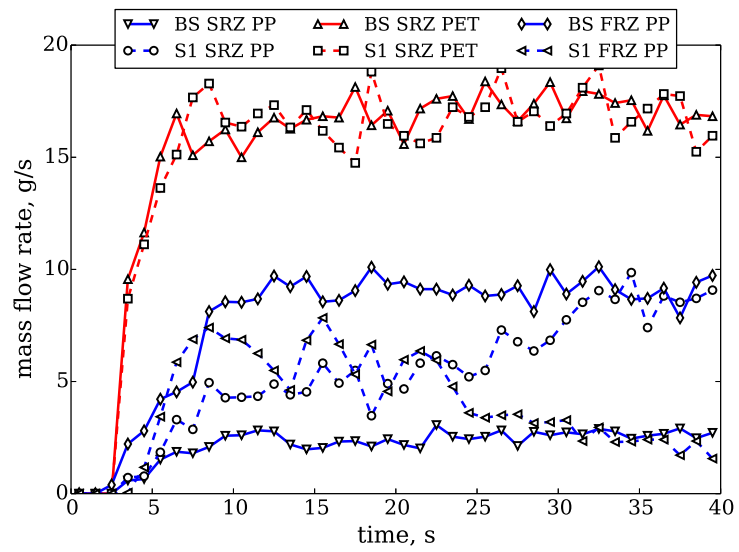

Figure 10: The mass flow rate of particle removal for $\mathrm{S} 1$ and BS simulations

curve), therefore the mass flow rate of PP particles entering the FRZ decreases ("S1 FRZ PP" curve). From the performed simulation it can be concluded, that the use of the separating vertical walls helps to reduce the pollution of the discharged PET particles with PP particles, which makes the use of the separating vertical walls a preferable design solution.

\subsubsection{Influence of the rotational velocity of the drum}

A numerical analysis of the influence of the rotational velocity of the drum is carried out. In addition to the $\mathrm{BS}$ simulation, where the drum rotated at $0.5 \pi \mathrm{rad} / \mathrm{s}$ velocity, two simulations, where the drum rotates at $0.25 \pi \mathrm{rad} / \mathrm{s}(\mathrm{sim}-$ ulation S2 in Table 1) and at $1.0 \pi \mathrm{rad} / \mathrm{s}$ (S3), are performed. The mass flow rate of the resulting particle removal is shown in Fig.[11. In all three simulations the mass flow rate of PET particles removed through the SRZ is at the same level, however in the $\mathrm{S} 2$ case (lowest rotational velocity) this level is reached a bit later than in the BS and S3 simulations. The "BS SRZ PP", "S2 SRZ PP" and "S3 SRZ PP" curves show how many PP particles were lifted and removed together with PET particles into the SRZ. In the S3 case more PP particles are trapped 


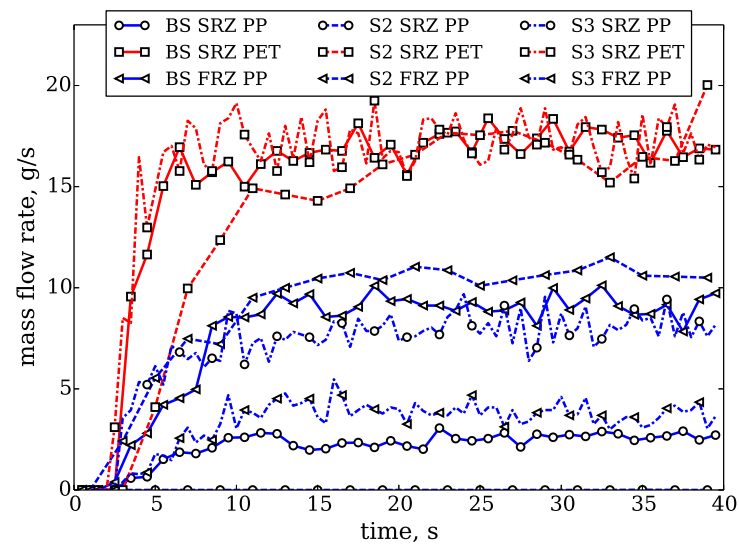

Figure 11: Influence of the rotational velocity of the drum: the mass flow rate of particle removal

together with PET particles ("S3 SRZ PP" curve) than flow-out through the opening together with the water ("S3 FRZ PP" curve). A completely different situation can be seen in the S2 simulation: just very few PP particles are lifted into the SRZ and therefore the "S2 SRZ PP" curve always remains at zero level. This is observed because, when the drum rotates more slowly, the PP and PET particles have more time to separate from each other and PP particles are not caught together with PET particles. The "BS SRZ PP" curve remains at an intermediate level between corresponding S2 and S3 results. It can be concluded, that the rotational drum velocity has a big influence on the resulting purity of the separated particles.

\subsubsection{Influence of the number of lifters}

In the BS simulation 4 lifters (see Fig.6) on the sides of the drum are used. Simulations with 2 (S4) and 8 (S5) lifters are performed additionally. The results are presented in Fig.12, "BS SRZ PET" and "S5 SRZ PET" curves are keeping on the same level, however the corresponding curve from the S4 simulation, where only 2 lifters are used, is reaching this level only at about $35 \mathrm{~s}$. This indicates, that in the $\mathrm{S} 4$ simulation more PET particles are remaining inside of the drum. Comparing the "BS SRZ PP", "S4 SRZ PP" and "S5 SRZ PP" curves is clear, that the lowest mass rate of $\mathrm{PP}$ particles removed together with PET is in the case when 8 lifters are used. "S5 FRZ PP" has a tendency to decrease during the second part of the simulation, which indicates, that more $\mathrm{PP}$ particles are remaining in the drum.

\subsubsection{Influence of the feed rate of solid particles}

The influence of the feed rate of solid particles to the sorting process is analysed. Simulations with three different feed rates of solid particles are performed: BS with a feed rate of $29.3 \mathrm{~g} / \mathrm{s}$, S6 with a two times lower feed rate of $14.65 \mathrm{~g} / \mathrm{s}$ and $\mathrm{S} 7$ with a two times higher feed rate of $58.6 \mathrm{~g} / \mathrm{s}$. The results of the simulations are presented in Fig.13. Comparing the "BS FRZ PP", "S6 FRZ PP" and "S7 FRZ PP" curves it can be seen, that the character of these curves is similar, however the level of the mass flow rate indicates the differences in the 


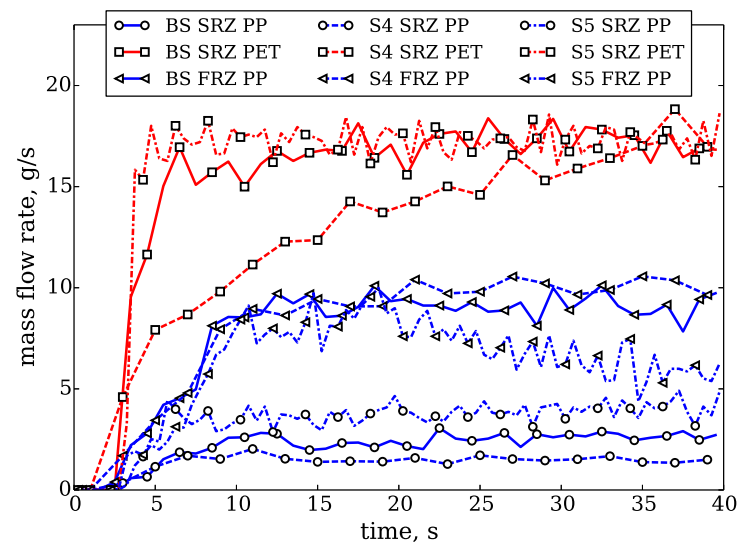

Figure 12: Influence of the number of lifters in the drum: the mass rate of particle removal

feed rate: the curve "S6 FRZ PP" keeps at about $4.5 \mathrm{~g} / \mathrm{s}$, the curve "BS FRZ PP" keeps at about $9.0 \mathrm{~g} / \mathrm{s}$, while the curve "S7 FRZ PP" keeps at about $18.0 \mathrm{~g} / \mathrm{s}$. The influence on the PET particles coming to the SRZ can be seen from the comparison of the "BS SRZ PET", "S6 SRZ PET" and "S7 SRZ PET" curves. While the "BS SRZ PET" and "S6 SRZ PET" curves are keeping at a constant level from about $6 \mathrm{~s}$, the "S7 SRZ PET" curve is increasing until the end of the simulation. This is because the part of the lifter near to the particle feed zone is fully loaded and therefore cannot lift all sunken PET particles in simulation S7 as can be seen in Fig.14. With the time the amount of sunken PET particles in the drum is increasing, and the zone with the sunken PET particles is increasing too. Therefore, the bigger part of the lifter is used to lift the particles, which is resulting in the increase of the rate of the removed PET particles ("S7 SRZ PET" curve). The differences in the "BS SRZ PP", "S6 SRZ PP" and "S7 SRZ PP" curves indicates, that the amount of PP particles removed together with the sunken PET particles is increasing when more particles are fed into the drum. However, the increase of these removed PP particles corresponds to the increase of the amount of the removed PET particles.

\subsubsection{Influence of the water feed rate}

The mixture of solid particles can be fed into the drum with different amounts of water. The influence of the water feed rate is analysed by performing three simulations with water feed rates $1.035 \mathrm{l} / \mathrm{s}, 2.07 \mathrm{l} / \mathrm{s}$ and $4.14 \mathrm{l} / \mathrm{s}$. The results of these simulations are presented in Fig.15. The "BS SRZ PET", "S8 SRZ PET" and "S9 SRZ PET" curves are at the same level, which indicates that the amount of water makes no influence on the settling and the removal of the PET particles. The small influence of the amount of the water can be observed by comparing the "BS SRZ PP", "S8 SRZ PP" and "S9 SRZ PP" curves. It can be seen, that the "S9 SRZ PP" curve is below the other two curves, which indicates, that in S9 a bit less PP particles are caught together with the settled PET particles. 


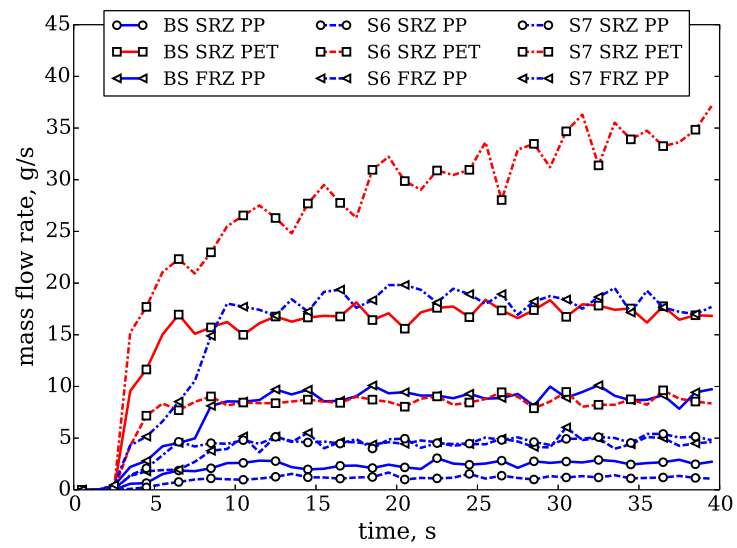

Figure 13: The mass flow rate of particle removal: Influence of the feed rate of solid particles
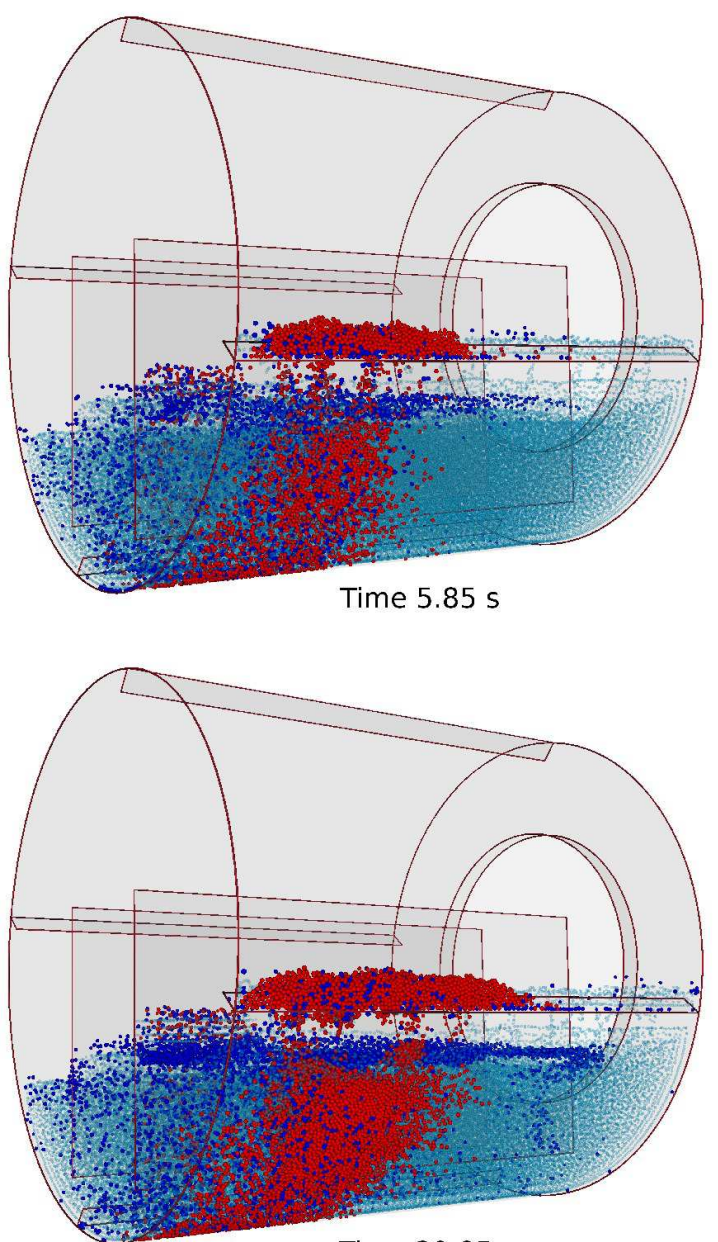

Time $39.85 \mathrm{~s}$

Figure 14: Snapshots of the simulation S7 


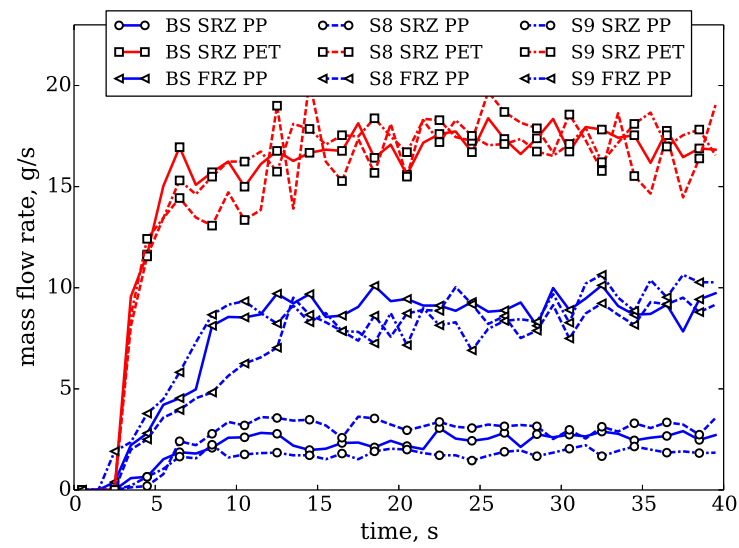

Figure 15: The mass flow rate of particle removal: Influence of the feed rate by solids

\subsubsection{Comparison of resultant purity of PET}

The resultant PET purity obtained in the simulations is summarised in Fig.16. Here, the purity is defined as the mass of PET particles removed in the SRZ divided by the mass of all particles which were removed in the SRZ:

$$
\text { Purity }_{\mathrm{PET}}=\frac{\text { masspeT,SRZ }}{(\operatorname{mass} \mathrm{PET}, \mathrm{SRZ}+\operatorname{mass} \mathrm{PP}, \mathrm{SRZ})} .
$$

As can be seen in Fig.16, the resultant purity for the basic setup (BS) is equal to $87.6 \%$. The removal of the vertical walls (S1) reduces the purity to $74.0 \%$. With the lowering of the rotational velocity of the drum the purity of PET increases up to $100.0 \%$, while the increase of rotational velocity reduces the purity down to $69.0 \%$. The reduction of the number of used lifters from 4 to 2 (BS and S4 cases), increases the purity to $90.3 \%$, while the increase of the number of the lifters ( 8 lifters in S5 case) decrease the purity to $82.3 \%$. It was found, that in the tested range the feed rate of solid particles has just a small influence on the resultant purity (S6 and S7 cases). The purity of PET increased from $84.8 \%$ to $90.6 \%$, when the feed rate by water was increased from $1.035 \mathrm{l} / \mathrm{s}$ up to $4.14 \mathrm{l} / \mathrm{s}$ (S8 and S9 cases). It could be concluded, that from all tested parameters, the rotational velocity has the biggest influence on the resultant purity of PET.

\section{Conclusions}

In the present study, an application of a coupled DEM-SPH scheme for the analysis of wet plastic particle separation was presented. The used DEM-SPH scheme was described and dam break tests were performed. The results were compared with published results found in literature, which, together with our earlier study [28], let us conclude about the validity of the used technique. The numerical analysis of polyethylene terephthalate (PET) particle separation from polypropylene (PP) particles in a rotating drum was performed. The influence of different operational and design parameters, such as the rotational velocity, the number of lifters, the feed rate etc., was analysed. Numerical results show, that the use of the separating vertical walls, lower rotational velocity, 


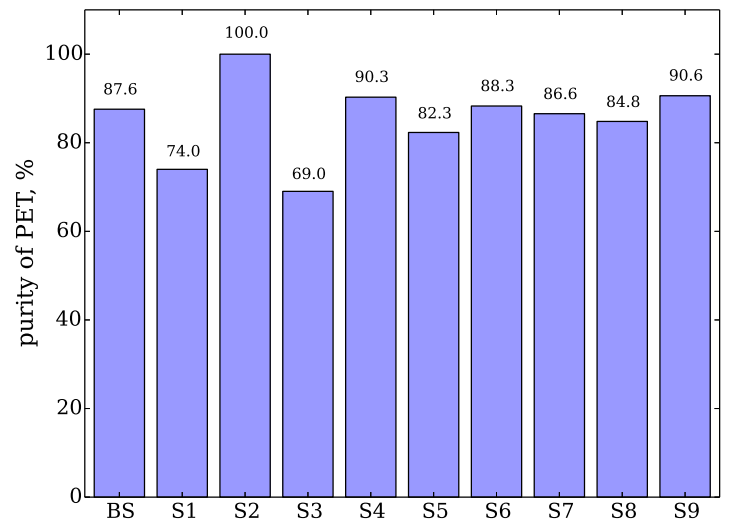

Figure 16: Purity of PET

higher number of lifters and a higher water feed rate increases the purity of the separated particles. While the used technique was validated by comparing the simulation results of dam break problem with published experimental data, a direct comparison of the numerical simulation of the drum separation process is a desirable and logical next step in the future.

\section{Acknowledgements}

This project has received funding from the European Union's Horizon 2020 research and innovation programme under the Marie Sklodowska-Curie grant agreement No. 652862 .

\section{References}

[1] G. Dodbiba, N. Haruki, a. Shibayama, T. Miyazaki, T. Fujita, Combination of sink-float separation and flotation technique for purification of shredded PET-bottle from PE or PP flakes, International Journal of mineral processing 65 (2002) 11-29.

[2] C. Delgado, A. Stenmark, Technological Reference Paper on Recycling Plastics, Tech. rep., Virtual European Recycling Centre (2005).

[3] J. Brandrup, Recycling and Recovery of Plastics, Hanser Publishers, 1996.

[4] R. Sripriya, a. Dutta, P. K. Dhall, M. Narasimha, V. Kumar, B. S. Tiwari, An analysis of medium losses in coal washing plants, International Journal of Mineral Processing 80 (2-4) (2006) 177-188.

[5] G. Dodbiba, T. Fujita, Progress in separating plastic materials for recycling, Physical Separation in Science and Engineering 13 (3-4) (2004) 165-182.

[6] S. Pongstabodee, N. Kunachitpimol, S. Damronglerd, Combination of three-stage sink-float method and selective flotation technique for separation of mixed post-consumer plastic waste, Waste Management 28 (3) (2008) 475-483. 
[7] N. Menad, S. Guignot, J. A. van Houwelingen, New characterisation method of electrical and electronic equipment wastes ( WEEE ), Waste Management 33 (3) (2013) 706-713.

[8] H. Zhu, Z. Zhou, R. Yang, A. Yu, Discrete particle simulation of particulate systems: A review of major applications and findings, Chemical Engineering Science 63 (23) (2008) 5728-5770.

[9] W. Zhong, A. Yu, X. Liu, Z. Tong, H. Zhang, DEM / CFD-DEM Modelling of Non-spherical Particulate Systems : Theoretical Developments and Applications, Powder Technology 302 (2016) 108-152.

[10] K. Han, Y. Feng, D. Owen, Coupled lattice Boltzmann and discrete element modelling of fluid-particle interaction problems, Computers \& Structures 85 (11-14) (2007) 1080-1088.

[11] T. B. Anderson, R. Jackson, Fluid mechanical description of fluidized beds. Equations of motion, Industrial \& Engineering Chemistry Fundamentals 6 (4) (1967) 527-539.

[12] H. Kruggel-Emden, B. Kravets, M. K. Suryanarayana, R. Jasevicius, Direct numerical simulation of coupled fluid flow and heat transfer for single particles and particle packings by a LBM-approach, Powder Technology 294 (2016) 236-251.

[13] L. Wang, G. Zhou, X. Wang, Q. Xiong, W. Ge, Direct numerical simulation of particle-fluid systems by combining time-driven hard-sphere model and lattice Boltzmann method, Particuology 8 (4) (2010) 379-382.

[14] F. Zhao, B. G. M. Van Wachem, Direct numerical simulation of ellipsoidal particles in turbulent channel flow, Acta Mechanica 224 (10) (2013) 23312358.

[15] J. Ferziger, M. Peric, Computational Methods for Fluid Dynamics, 3rd Edition, Springer, Berlin, 2002.

[16] D. Gao, J. a. Herbst, Alternative ways of coupling particle behaviour with fluid dynamics in mineral processing, International Journal of Computational Fluid Dynamics 23 (2) (2009) 109-118.

[17] R. A. Gingold, J. J. Monaghan, Smoothed particle hydrodynamics: theory and application to non-spherical stars, Monthly Notices of the Royal Astronomical Society 181 (3) (1977) 375-389.

[18] L. Lucy, A numerical approach to the testing of the fission hypothesis, Astron. J. 82 (1977) 1013-1024.

[19] K. Shibata, S. Koshizuka, Numerical analysis of shipping water impact on a deck using a particle method, Ocean Engineering 34 (3-4) (2007) 585-593.

[20] M. Prakash, P. W. Cleary, Modelling highly deformable metal extrusion using SPH, Computational Particle Mechanics 2 (1) (2015) 19-38. 
[21] R. Sivanesapillai, H. Steeb, A. Hartmaier, Transition of effective hydraulic properties from low to high reynolds number flow in porous media, Geophysical Research Letters 41 (14) (2014) 4920-4928.

[22] H. Gotoh, T. Sakai, Key issues in the particle method for computation of wave breaking, Coastal Engineering 53 (2006) 171-179.

[23] X. Sun, M. Sakai, Y. Yamada, Three-dimensional simulation of a solidliquid flow by the DEM-SPH method, Journal of Computational Physics 248 (2013) 147-176.

[24] M. Robinson, M. Ramaioli, S. Luding, Fluid-particle flow simulations using two-way-coupled mesoscale SPH-DEM and validation, International Journal of Multiphase Flow 59 (2014) 121-134.

[25] P. W. Cleary, Prediction of coupled particle and fluid flows using DEM and SPH, Minerals Engineering 73 (2015) 85-99.

[26] F. Beck, P. Eberhard, Predicting abrasive wear with coupled Lagrangian methods, Computational Particle Mechanics 2 (1) (2015) 51-62.

[27] H. G. Lagger, T. Breinlinger, J. G. Korvink, M. Moseler, A. D. Renzo, F. D. Maio, C. Bierwisch, Influence of hydrodynamic drag model on shear stress in the simulation of magnetorheological fluids, Journal of Non-Newtonian Fluid Mechanics 218 (2015) 16-26.

[28] D. Markauskas, H. Kruggel-emden, R. Sivanesapillai, H. Steeb, Comparative study on mesh-based and mesh-less coupled CFD-DEM methods to model particle-laden flow, Powder Technology 305 (2017) 78-88.

[29] I. T. Tsuji Y., Tanaka T., Lagrangian numerical simulation of plug flow of cohesionless particles in a horizontal pipe, Powder Technology 71 (1992) 239-250.

[30] A. Dziugys, B. Peters, An approach to simulate the motion of spherical and non-spherical fuel particles in combustion chambers, Granular Matter 3 (2001) 231-265.

[31] H. Kruggel-Emden, T. Oschmann, Numerical study of rope formation and dispersion of non-spherical particles during pneumatic conveying in a pipe bend, Powder Technology 268 (2014) 219-236.

[32] D. Markauskas, A. Kačeniauskas, The comparison of two domain repartitioning methods used for parallel discrete element computations of the hopper discharge, Advances in Engineering Software 84 (2015) 68-76.

[33] J. J. Monaghan, Smoothed particle hydrodynamics, Reports on Progress in Physics 68 (8) (2005) 1703-1759.

[34] J. Monaghan, R. Gingold, Shock simulation by the particle method SPH, Journal of Computational Physics 52 (2) (1983) 374-389.

[35] R. Dalrymple, B. Rogers, Numerical modeling of water waves with the SPH method, Coastal Engineering 53 (2-3) (2006) 141-147. 
[36] H. Wendland, Piecewise polynomial, positive definite and compactly supported radial functions of minimal degree, Advances in Computational Mathematics 4 (1) (1995) 389-396.

[37] J. Monaghan, A. Kos, Solitary waves on a Cretan beach, Journal of Waterway Port Coastal and Ocean Engineering 125 (3) (1999) 145-155.

[38] M. Gómez-Gesteira, R. A. Dalrymple, Using a three-dimensional smoothed particle hydrodynamics method for wave impact on a tall structure, Journal of Waterway, Port, Coastal, and Ocean Engineering 130 (2) (2004) 63-69.

[39] A. Colagrossi, M. Landrini, Numerical simulation of interfacial flows by smoothed particle hydrodynamics, Journal of Computational Physics 191 (2) (2003) 448-475.

[40] J. P. Morris, P. J. Fox, Y. Zhu, Modeling low reynolds number incompressible flows using SPH, Journal of Computational Physics 136 (1997) $214-226$.

[41] S. Adami, X. Hu, N. Adams, A generalized wall boundary condition for smoothed particle hydrodynamics, Journal of Computational Physics 231 (21) (2012) 7057-7075.

[42] S. Marrone, a. Colagrossi, M. Antuono, G. Colicchio, G. Graziani, An accurate SPH modeling of viscous flows around bodies at low and moderate Reynolds numbers, Journal of Computational Physics 245 (2013) 456-475.

[43] A. Valizadeh, J. J. Monaghan, A study of solid wall models for weakly compressible SPH, Journal of Computational Physics 300 (2015) 5-19.

[44] J. Monaghan, Simulating free surface flows with sph, Journal of Computational Physics 110 (2) (1994) 399-406.

[45] R. Di Felice, The voidage function for fluid-particle interaction systems, International Journal of Multiphase Flow 20 (1) (1994) 153-159.

[46] T. Oschmann, J. Hold, H. Kruggel-Emden, Numerical investigation of mixing and orientation of non-spherical particles in a model type fluidized bed, Powder Technology 258 (2014) 304-323.

[47] C. W. Hirt, B. D. Nichols, Volume of Fluid ( VOF ) Method for the Dynamics of Free Boundaries, Journal of Computational Physics 39 (1981) $201-225$. 